Supporting Informations for the Communication

Entitled

\title{
Heavy Ferrocene: A Sandwich Complex Containing Si and Ge Atoms
}

\author{
Vladimir Ya. Lee, Risa Kato, and Akira Sekiguchi* \\ Department of Chemistry, Graduate School of Pure and Applied Sciences, \\ University of Tsukuba, Tsukuba, Ibaraki 305-8571, Japan
}

\begin{abstract}
Andreas Krapp and Gernot Frenking*
Fachbereich Chemie, Philipps-Universität Marburg, Hans-Meerwein-Strasse D-35032 Marburg, Germany
\end{abstract}


Experimental Section

General Procedurc. All experiments were performed using high-vacuum line techniques or in an argon atmosphere of MBRAUN MB 150B-G glove-box. All solvents were predried over sodium benzophenone ketyl and finally dried and degassed over potassium mirror in vacuo prior to use. UV-spectra were recorded on a Shimadzu UV-3150 UV-Vis spectrophotometer in hexane.

\section{Experimental Procedure and Spectral Data for $\left(\eta^{5}-1,2,3-\operatorname{tris}(\right.$ di-tert-butylmethylsilyl)-4-} phenyl-1,2-disila-3-germacyclopentadienyl $)\left(\eta^{5}\right.$-pentamethylcyclopentadienyl)iron (2) and Crystallographic Data of 2.

A mixture of $\mathrm{Fe}(\mathrm{acac})_{2}(55 \mathrm{mg}, 0.22 \mathrm{mmol})$ and $\mathrm{Cp} * \mathrm{Li}\left(\mathrm{Cp}^{*}=\mathrm{C}_{5}\left(\mathrm{CH}_{3}\right)_{5}\right)(32 \mathrm{mg}, 0.22$ mmol) was placed in a glass tube with a magnetic stirring bar in a glove-box, then dry oxygen-free THF $(2.0 \mathrm{ml})$ was transferred into this tube through a vacuum line, and the tube was sealed under vacuum. After stirring for 15 minutes at $-78^{\circ} \mathrm{C}$, the cooling bath was removed, and the mixture was warmed to room temperature. The color of the reaction mixture turned to dark yellowish red, and the resulting $\mathrm{Li}(\mathrm{acac})$ was precipitated. The THF solution of [Cp*Fe(acac)] and lithium 1,2,3-tris(di-tert-butylmethylsilyl)-4-phenyl-1,2-disila-3-germacyclopentadienide $\left(1^{-} \cdot\left[\mathrm{Li}^{+}(\mathrm{thf})\right]\right)[1](166 \mathrm{mg}, 0.21 \mathrm{mmol})$ were mixed at $-78^{\circ} \mathrm{C}$, and the resulting reaction mixture was stirred for 15 minutes at low temperature and 30 minutes at room temperature. After evaporation of solvent, the residue was dissolved in hexane $(2.0 \mathrm{ml})$, and the side product, Li(acac), was removed by centrifugation. The target iron complex 2 was separated by silica gel column chromatography (eluent: hexane) in a glove box, followed by recrystallization from pentane, and was isolated in a pure form as dark red crystals $(58 \mathrm{mg}, 31 \%)$; m.p. $203-205^{\circ} \mathrm{C}$; UV/Vis (hexane) $\lambda_{\max } / \mathrm{nm}(\varepsilon) 228$ (51100), 316 (16500), 396 (4080), 489 (1240); ${ }^{1} \mathrm{H}$ NMR (400 
MHz, $\left.\mathrm{C}_{6} \mathrm{D}_{6}, \delta\right) 0.54(\mathrm{~s}, 3 \mathrm{H}, \mathrm{Me}), 0.59(\mathrm{~s}, 3 \mathrm{H}, \mathrm{Me}), 0.74(\mathrm{~s}, 3 \mathrm{H}, \mathrm{Me}), 1.03\left(\mathrm{~s}, 9 \mathrm{H},{ }^{\prime} \mathrm{Bu}\right), 1.20(\mathrm{~s}$, $\left.9 \mathrm{H},{ }^{\prime} \mathrm{Bu}\right), 1.26\left(\mathrm{~s}, 9 \mathrm{H},{ }^{\prime} \mathrm{Bu}\right), 1.27$ (s, $\left.9 \mathrm{H},{ }^{\prime} \mathrm{Bu}\right), 1.31\left(\mathrm{~s}, 9 \mathrm{H},{ }^{\prime} \mathrm{Bu}\right), 1.35\left(\mathrm{~s}, 9 \mathrm{H},{ }^{\prime} \mathrm{Bu}\right), 1.91(\mathrm{~s}, 15 \mathrm{H}$, $\left.\mathrm{C}_{5} \mathrm{Me}_{5}\right), 4.49$ (s, $\left.1 \mathrm{H}, \mathrm{C}=\mathrm{CH}\right), 7.10$ (t, $\left.1 \mathrm{H}, J=7.4 \mathrm{~Hz}, \mathrm{H}_{\mathrm{para}}\right), 7.22$ (t, $\left.2 \mathrm{H}, J=7.4 \mathrm{~Hz}, \mathrm{H}_{\text {meta }}\right), 7.94$ (d, $\left.2 \mathrm{H}, J=7.4 \mathrm{~Hz}, \mathrm{H}_{\text {ortho }}\right) ;{ }^{13} \mathrm{C}$ NMR $\left(100.6 \mathrm{MHz}, \mathrm{C}_{6} \mathrm{D}_{6}, \delta\right)-2.8(\mathrm{Me}),-1.19(\mathrm{Me}),-1.15(\mathrm{Me})$, $14.0\left(\mathrm{C}_{5} \mathrm{Me}_{5}\right), 21.2\left(\mathrm{CMe}_{3}\right), 21.3\left(\mathrm{CMe}_{3}\right), 21.5\left(\mathrm{CMe}_{3}\right), 22.2\left(\mathrm{CMe}_{3}\right), 22.3\left(\mathrm{CMe}_{3}\right), 22.8\left(\mathrm{CMe}_{3}\right)$, $29.4\left(\mathrm{CMe}_{3}\right), 29.9\left(\mathrm{CMe}_{3}\right), 30.6\left(\mathrm{CMe}_{3}\right), 30.7\left(\mathrm{CMe}_{3}\right), 30.9\left(\mathrm{CMe}_{3}\right), 31.0\left(\mathrm{CMe}_{3}\right), 80.5(\mathrm{C}=\mathrm{CH}$, $\left.{ }^{1} J\left({ }^{13} \mathrm{C}^{1} \mathrm{H}\right)=143 \mathrm{~Hz}\right), 84.2\left(C_{5} \mathrm{Me}_{5}\right), 108.1(\mathrm{PhC}=\mathrm{C}), 124.7\left(\mathrm{C}_{\text {arom }}\right), 127.3\left(\mathrm{C}_{\text {arom }}\right), 131.7\left(\mathrm{C}_{\text {arom }}\right)$,

$151.1\left(\mathrm{C}_{\mathrm{ipso}}\right)$; ${ }^{29} \mathrm{Si} \mathrm{NMR}(79.5 \mathrm{MHz}, \delta)-49.3,1.1,26.5,29.4,33.4$; Anal. Found. C, 60.01: H, 9.64. Calc. for $\mathrm{C}_{45} \mathrm{H}_{84} \mathrm{FeGeSi}$ : $\mathrm{C}, 60.45: \mathrm{H}, 9.47 \%$.

The single crystals of $\mathbf{2}$ for $\mathrm{X}$-ray diffraction analysis were grown from a saturated pentane solution. Diffraction data were collected at $120 \mathrm{~K}$ on a Mac Science DIP2030 Image Plate Diffractometer with a rotating anode $(50 \mathrm{kV}, 90 \mathrm{~mA})$ employing graphite-monochromatized Mo-Ka radiation $(\lambda=0.71070 \AA)$. The structure was solved by the direct method using SIR-92 [2] program and refined by the full-matrix least-squares method by SHELXL-97 program [3]. Crystal data for 2 at $120 \mathrm{~K}: \mathrm{MF}=\mathrm{C}_{45} \mathrm{H}_{84} \mathrm{FeGeSi}_{5}, \mathrm{MW}=894.01$, triclinic, $P-1, a=9.9320(5), b$ $=11.9290(6), c=21.9830(6) \AA, a=82.796(3), b=87.779(3), g=81.784(2)^{\circ}, V=2556.86(19)$ $\AA^{3}, \quad Z=2, D_{\text {calcd }}=1.161 \mathrm{~g} \cdot \mathrm{cm}^{-3}$. The final $R$ factor was 0.0524 for 8706 reflections with $I_{0}>$ $2 \sigma\left(I_{0}\right)\left(R_{\mathrm{w}}=0.1567\right.$ for all data, 11427 reflections $), \mathrm{GOF}=1.034$.

\section{Computational Details}

The geometries of the complexes were optimized using the TURBOMOLE 5.9 program [4]. All results are obtained from Kohn-Sham calculations. We employed the Becke-Perdew functional BP86 $[5,6]$ as implemented in the Turbomole program. In connection with the BP86 functional we always used the resolution of the identity (RI) technique $[7,8]$. We used the triple- $\zeta$ 
basis sets of Weigend and Ahlrichs named def2-TZVPP [9] in conjunction with the accompanying auxiliary basis sets for the Coulomb fitting in the RI-DFT calculations as implemented in Turbomole [10]. The nature of the stationary points as true minima was verified by analytically calculating the Hessian matrix and inspection of its eigenvalues.

The energy decomposition analysis (EDA) was performed with the ADF2005 program [11] on the RI-BP86/def2-TZVPP optimized structures at the BP86 level using uncontracted Slater-type orbitals (STOs) which have TZ2P quality [12]. The focus of the EDA bonding analysis [13] is the instantaneous interaction energy $\Delta \mathrm{E}_{\mathrm{int}}$, which is the energy difference between the molecule and the fragments with the frozen geometry of the complex. The interaction energy is divided into three main components:

$$
\Delta E_{\text {int }}=\Delta E_{\text {elstat }}+\Delta E_{\text {Pauli }}+\Delta E_{\text {orb }}
$$

The term $\Delta E_{\text {elstat }}$ gives the electrostatic interaction energy between the fragments, which are calculated with a frozen density distribution in the geometry of the complex. The Pauli repulsion $\left(\Delta E_{\text {Pauli }}\right)$ arises as the energy change associated with the transformation from the superposition of the unperturbed electron densities of fragments $\rho_{A}+\rho_{B}$ to the wavefunction $\Psi^{0}=N \hat{A}\left\{\Psi_{A} \bullet \Psi_{B}\right\}$, which properly obeys the Pauli principle through explicit antisymmetrisation $(\hat{\mathrm{A}})$ and renormalisation $(N)$ of the product wavefunction. It comprises the destabilizing interactions between electrons on either fragment with the same spin. The stabilizing orbital interaction term $\Delta E_{\text {orb }}$ is calculated in the final step of the analysis when the orbitals relax to their final form. The latter can be decomposed into contributions from each irreducible representation of the point group of the interacting system. In order to obtain the bond dissociation energy (BDE) one has to consider the preparation energy $\Delta E_{\text {prep}}$, which is the energy difference of the fragments between their equilibrium geometry and their geometry in the molecule:

$$
\Delta E(=-\mathrm{BDE})=\Delta E_{\mathrm{int}}+\Delta E_{\mathrm{prep}}
$$




\section{References}

[1] Lee, V. Ya.; Kato, R.; Ichinohe, M.; Sekiguchi, A. J. Am. Chem. Soc. 2005, 127, 13142.

[2] Altomare, A.; Cascarano, G.; Giacovazzo, C.; Guagliardi, A.; Burla, M. C.; Polidori, G.; Camalli, M. J. Appl. Crystallogr. 1994, 27, 435.

[3] Sheldrick, G. M. SHELXL-97, Program for Crystal Structure Refinement, University of Göttingen, Germany, 1997.

[4] Ahlrichs, R.; Bär, M.; Häser, M.; Horn, H.; Kölmel, C. Chem. Phys. Lett. 1989, 162, 165.

[5] Becke, A. D. Phys. Rev. A 1988, 38, 3098.

[6] Perdew, J. P. Phys. Rev. B 1986, 33, 8822.

[7] Eichkorn, K.; Treutler, O.; Öhm, H.; Häser, M.; Ahlrichs, R. Chem. Phys. Lett. 1995, 240, 283.

[8] Eichkorn, K.; Weigend, F.; Treutler, O.; Ahlrichs, R. Theor: Chem. Acc. 1997, 97, 119.

[9] Weigend, F.; Ahlrichs, R. Phys. Chem. Chem. Phys. 2005, 7, 3297.

[10] Weigend, F. Phys. Chem. Chem. Phys. 2006, 8, 1057.

[11] (a) ADF2005, SCM, Theoretical Chemistry, Vrije Universiteit, Amsterdam, The Netherlands, http://www.scm.com (b) Bickelhaupt, F. M.; Baerends, E. J. Rev. Comput. Chem. Vol. 15, p. 1, Lipkowitz, K. B.; Boyd, D. B. (Eds.), Wiley-VCH, New York, 2000; (c) te Velde, G.; Bickelhaupt, F. M.; Baerends, E. J.; van Gisbergen, S. J. A.; Fonseca Guerra, C.; Snijders, J. G.; Ziegler, T. J. Comput. Chem. 2001, 22, 931.

[12] Snijders, J. G.; Baerends, E. J.; Vernooijs, P. At. Nucl. Data Tables 1982, 26, 483.

[13] For reviews about the application of the EDA, see reference $11 \mathrm{~b}$ and the following: (a) Lein, M.; Frenking, G. In Theory and Applications of Computational Chemistry: The First 40 Years, p. 367, Dykstra, C. E.; Frenking, G.; Kim, K. S.; Scuseria, G. E. (Eds.), Elsevier, 
Amsterdam, 2005. (b) Frenking, G.; Wichmann, K.; Fröhlich, N.; Loschen, C.; Lein, M. Frunzke, J.; Rayón, V. M. Coord. Chem. Rev. 2003, 238-239, 55. 
Table 1. Crystal data and structure refinement for 2 .

\begin{tabular}{|c|c|}
\hline Identification code & shelxl \\
\hline Empirical formula & C45 H84 Fe Ge Si5 \\
\hline Formula weight & 894.01 \\
\hline Temperature & $120 \mathrm{~K}$ \\
\hline Wavelength & $0.71070 \mathrm{~A}$ \\
\hline Crystal system, space group & Triclinic, $\quad P-1$ \\
\hline Unit cell dimensions & 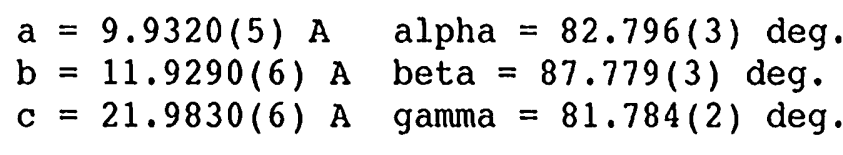 \\
\hline Volume & $2556.86(19) A^{\wedge} 3$ \\
\hline Z, Calculated density & $2, \quad 1.161 \mathrm{Mg} / \mathrm{m}^{\wedge} 3$ \\
\hline Absorption coefficient & $1.016 \mathrm{~mm}^{\wedge}-1$ \\
\hline$F(000)$ & 964 \\
\hline Crystal size & $0.50 \times 0.30 \times 0.20 \mathrm{~mm}$ \\
\hline Theta range for data collection & 2.39 to $28.02 \mathrm{deg}$. \\
\hline Limiting indices & $0<=h<=13,-15<=k<=15,-28<=1<=28$ \\
\hline Reflections collected / unique & $11427 / 11427[R($ int $)=0.042]$ \\
\hline Completeness to theta $=28.02$ & $92.5 \div$ \\
\hline Absorption correction & None \\
\hline Refinement method & Full-matrix least-squares on $F^{\wedge} 2$ \\
\hline Data / restraints / parameters & $11427 / 0 / 470$ \\
\hline Goodness-of-fit on $F^{\wedge} 2$ & 1.034 \\
\hline Final $R$ indices $[I>2 \operatorname{sigma}(I)]$ & $\mathrm{R} 1=0.0524, \mathrm{WR} 2=0.1461$ \\
\hline$R$ indices (all data) & $\mathrm{R} 1=0.0702, \mathrm{wR} 2=0.1567$ \\
\hline Extinction coefficient & $0.0103(11)$ \\
\hline
\end{tabular}




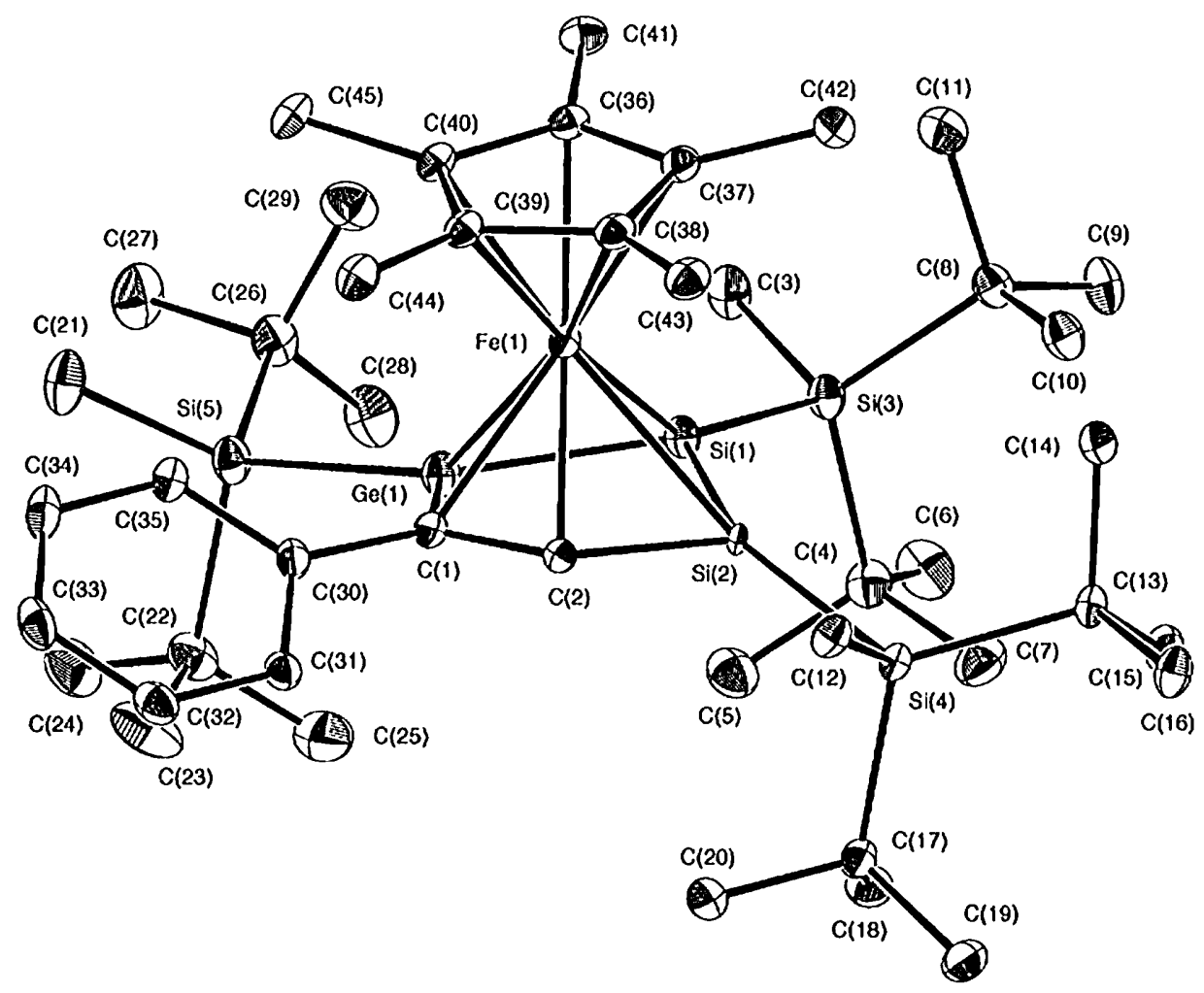

Figure 1. ORTEP drawing of ( $\eta^{5}-1,2,3$-tris(di-tert-butylmethylsilyl)-4-phenyl1,2-disila-3-germacyclopentadienyl)( $\eta^{5}$-pentamethylcyclopentadienyl)iron (2) 
Table 2. Atomic coordinates $\left(\times 10^{\wedge} 4\right)$ and equivalent isotropic displacement parameters $\left(A^{\wedge} 2 \times 10^{\wedge} 3\right)$ for 2 .

$\mathrm{U}(\mathrm{eq})$ is defined as one third of the trace of the orthogonalized Uij tensor.

\begin{tabular}{lrrrr}
\hline & & & & \\
& \multicolumn{1}{c}{$\mathrm{x}$} & $\mathrm{y}$ & $\mathrm{U}(\mathrm{eq})$ \\
\hline $\mathrm{Ge}(1)$ & $1470(1)$ & $6361(1)$ & $2117(1)$ & $25(1)$ \\
$\mathrm{Fe}(1)$ & $2751(1)$ & $6177(1)$ & $3105(1)$ & $17(1)$ \\
$\mathrm{Si}(1)$ & $3069(1)$ & $4782(1)$ & $2354(1)$ & $22(1)$ \\
$\mathrm{Si}(2)$ & $2164(1)$ & $4219(1)$ & $3275(1)$ & $11(1)$ \\
$\mathrm{Si}(3)$ & $4548(1)$ & $3712(1)$ & $1691(1)$ & $24(1)$ \\
$\mathrm{Si}(4)$ & $1738(1)$ & $2811(1)$ & $4102(1)$ & $19(1)$ \\
$\mathrm{Si}(5)$ & $777(1)$ & $7728(1)$ & $1229(1)$ & $28(1)$ \\
$\mathrm{C}(1)$ & $596(3)$ & $6423(3)$ & $2911(1)$ & $20(1)$ \\
$\mathrm{C}(2)$ & $941(3)$ & $5486(2)$ & $3368(1)$ & $18(1)$ \\
$\mathrm{C}(3)$ & $5120(4)$ & $4650(3)$ & $1005(2)$ & $35(1)$ \\
$\mathrm{C}(4)$ & $3492(4)$ & $2648(3)$ & $1403(2)$ & $31(1)$ \\
$\mathrm{C}(5)$ & $2085(4)$ & $3268(4)$ & $1204(2)$ & $41(1)$ \\
$\mathrm{C}(6)$ & $4201(5)$ & $2154(4)$ & $839(2)$ & $45(1)$ \\
$\mathrm{C}(7)$ & $3290(5)$ & $1668(3)$ & $1906(2)$ & $41(1)$ \\
$\mathrm{C}(8)$ & $6193(3)$ & $3010(3)$ & $2097(2)$ & $28(1)$ \\
$\mathrm{C}(9)$ & $7024(4)$ & $2109(3)$ & $1735(2)$ & $36(1)$ \\
$\mathrm{C}(10)$ & $5909(4)$ & $2462(3)$ & $2750(2)$ & $32(1)$ \\
$\mathrm{C}(11)$ & $7087(4)$ & $3957(3)$ & $2134(2)$ & $37(1)$ \\
$\mathrm{C}(12)$ & $1228(4)$ & $3540(3)$ & $4809(1)$ & $26(1)$ \\
$\mathrm{C}(13)$ & $3226(3)$ & $1636(3)$ & $4328(1)$ & $24(1)$ \\
$\mathrm{C}(14)$ & $4485(4)$ & $2210(3)$ & $4420(2)$ & $31(1)$ \\
$\mathrm{C}(15)$ & $3562(4)$ & $825(3)$ & $3836(2)$ & $31(1)$ \\
$\mathrm{C}(16)$ & $2903(4)$ & $931(3)$ & $4939(2)$ & $32(1)$ \\
$\mathrm{C}(17)$ & $135(3)$ & $2289(3)$ & $3837(2)$ & $24(1)$ \\
$\mathrm{C}(18)$ & $298(4)$ & $1954(3)$ & $3180(2)$ & $30(1)$ \\
$\mathrm{C}(19)$ & $-248(4)$ & $1248(3)$ & $4272(2)$ & $33(1)$ \\
$\mathrm{C}(20)$ & $-1076(4)$ & $3253(3)$ & $3846(2)$ & $32(1)$ \\
$\mathrm{C}(21)$ & $328(5)$ & $9277(3)$ & $1358(2)$ & $42(1)$ \\
$\mathrm{C}(22)$ & $-854(4)$ & $7226(4)$ & $977(2)$ & $36(1)$ \\
$\mathrm{C}(23)$ & $-1942(4)$ & $7414(5)$ & $1477(2)$ & $57(1)$ \\
$\mathrm{C}(24)$ & $-1364(5)$ & $7931(4)$ & $370(2)$ & $52(1)$ \\
$\mathrm{C}(25)$ & $-667(5)$ & $5952(4)$ & $883(2)$ & $52(1)$ \\
$\mathrm{C}(26)$ & $2243(4)$ & $7712(3)$ & $633(2)$ & $34(1)$ \\
$\mathrm{C}(27)$ & $2016(5)$ & $8768(4)$ & $140(2)$ & $50(1)$ \\
$\mathrm{C}(28)$ & $2380(5)$ & $6647(4)$ & $299(2)$ & $45(1)$ \\
$\mathrm{C}(29)$ & $3572(4)$ & $7745(4)$ & $957(2)$ & $42(1)$ \\
$\mathrm{C}(30)$ & $-496(3)$ & $7348(3)$ & $3048(1)$ & $22(1)$ \\
$\mathrm{C}(31)$ & $-1612(3)$ & $7098(3)$ & $3429(1)$ & $23(1)$ \\
$\mathrm{C}(32)$ & $-2672(4)$ & $7939(3)$ & $3551(2)$ & $31(1)$ \\
$\mathrm{C}(33)$ & $-2642(4)$ & $9063(3)$ & $3301(2)$ & $34(1)$ \\
& & & &
\end{tabular}


S10

\begin{tabular}{lrlll}
$\mathrm{C}(34)$ & $-1562(4)$ & $9331(3)$ & $2926(2)$ & $33(1)$ \\
$\mathrm{C}(35)$ & $-495(4)$ & $8488(3)$ & $2804(2)$ & $28(1)$ \\
$\mathrm{C}(36)$ & $4525(3)$ & $6936(3)$ & $3005(1)$ & $22(1)$ \\
$\mathrm{C}(37)$ & $4612(3)$ & $6085(3)$ & $3537(1)$ & $23(1)$ \\
$\mathrm{C}(38)$ & $3506(3)$ & $6416(3)$ & $3949(1)$ & $23(1)$ \\
$\mathrm{C}(39)$ & $2760(3)$ & $7475(3)$ & $3679(1)$ & $22(1)$ \\
$\mathrm{C}(40)$ & $3393(3)$ & $7784(3)$ & $3099(1)$ & $22(1)$ \\
$\mathrm{C}(41)$ & $5529(4)$ & $7017(3)$ & $2479(2)$ & $28(1)$ \\
$\mathrm{C}(42)$ & $5731(4)$ & $5128(3)$ & $3687(2)$ & $29(1)$ \\
$\mathrm{C}(43)$ & $3261(4)$ & $5858(3)$ & $4581(2)$ & $29(1)$ \\
$\mathrm{C}(44)$ & $1609(4)$ & $8141(3)$ & $3998(2)$ & $29(1)$ \\
$\mathrm{C}(45)$ & $3096(4)$ & $8909(3)$ & $2696(2)$ & $29(1)$ \\
\hline
\end{tabular}


Table 3. Bond lengths [A] and angles [deg] for 2.

\begin{tabular}{|c|c|}
\hline $\mathrm{Ge}(1)-\mathrm{C}(1)$ & $1.924(3)$ \\
\hline $\mathrm{Ge}(1)-\mathrm{Si}(1)$ & $2.3038(9)$ \\
\hline $\mathrm{Ge}(1)-\mathrm{Si}(5)$ & $2.4343(9)$ \\
\hline $\mathrm{Ge}(1)-\mathrm{Fe}(1)$ & $2.5313(5)$ \\
\hline $\mathrm{Fe}(1)-\mathrm{C}(36)$ & $2.085(3)$ \\
\hline $\mathrm{Fe}(1)-\mathrm{C}(37)$ & $2.093(3)$ \\
\hline $\mathrm{Fe}(1)-\mathrm{C}(38)$ & $2.094(3)$ \\
\hline $\mathrm{Fe}(1)-\mathrm{C}(40)$ & $2.102(3)$ \\
\hline $\mathrm{Fe}(1)-\mathrm{C}(2)$ & $2.114(3)$ \\
\hline $\mathrm{Fe}(1)-\mathrm{C}(39)$ & $2.116(3)$ \\
\hline $\mathrm{Fe}(1)-\mathrm{C}(1)$ & $2.170(3)$ \\
\hline $\mathrm{Fe}(1)-\mathrm{Si}(2)$ & $2.4682(8)$ \\
\hline $\mathrm{Fe}(1)-\mathrm{Si}(1)$ & $2.4691(9)$ \\
\hline $\operatorname{Si}(1)-\operatorname{Si}(2)$ & $2.2465(11)$ \\
\hline $\operatorname{Si(1)-Si(3)}$ & $2.3835(12)$ \\
\hline $\operatorname{Si}(2)-C(2)$ & $1.827(3)$ \\
\hline $\operatorname{Si}(2)-\operatorname{Si}(4)$ & $2.3832(10)$ \\
\hline $\operatorname{Si}(3)-C(3)$ & $1.881(4)$ \\
\hline $\mathrm{Si}(3)-\mathrm{C}(8)$ & $1.924(4)$ \\
\hline $\operatorname{Si}(3)-C(4)$ & $1.934(4)$ \\
\hline $\operatorname{Si}(4)-C(12)$ & $1.894(3)$ \\
\hline $\operatorname{Si}(4)-C(13)$ & $1.919(3)$ \\
\hline $\mathrm{Si}(4)-\mathrm{C}(17)$ & $1.926(3)$ \\
\hline $\operatorname{Si}(5)-C(21)$ & $1.891(4)$ \\
\hline $\operatorname{Si}(5)-C(26)$ & $1.920(4)$ \\
\hline$S i(5)-C(22)$ & $1.929(4)$ \\
\hline$C(1)-C(2)$ & $1.419(4)$ \\
\hline$C(1)-C(30)$ & $1.484(4)$ \\
\hline$C(2)-H(2)$ & 0.9301 \\
\hline$C(3)-H(3 A)$ & 0.9600 \\
\hline$C(3)-H(3 B)$ & 0.9600 \\
\hline$C(3)-H(3 C)$ & 0.9600 \\
\hline$c(4)-c(7)$ & $1.535(5)$ \\
\hline$c(4)-C(5)$ & $1.538(5)$ \\
\hline$c(4)-c(6)$ & $1.542(5)$ \\
\hline$C(5)-H(5 A)$ & 0.9600 \\
\hline$C(5)-H(5 B)$ & 0.9600 \\
\hline $\mathrm{C}(5)-\mathrm{H}(5 \mathrm{C})$ & 0.9600 \\
\hline$C(6)-H(6 A)$ & 0.9600 \\
\hline$C(6)-H(6 B)$ & 0.9601 \\
\hline$C(6)-H(6 C)$ & 0.9600 \\
\hline$C(7)-H(7 A)$ & 0.9600 \\
\hline$C(7)-H(7 B)$ & 0.9600 \\
\hline $\mathrm{C}(7)-\mathrm{H}(7 \mathrm{C})$ & 0.9599 \\
\hline$C(8)-C(10)$ & $1.536(5)$ \\
\hline$C(8)-C(9)$ & $1.541(5)$ \\
\hline$C(8)-C(11)$ & $1.544(5)$ \\
\hline
\end{tabular}




\begin{tabular}{|c|c|}
\hline$C(9)-H(9 A)$ & 0.9601 \\
\hline$C(9)-H(9 B)$ & 0.9599 \\
\hline $\mathrm{C}(9)-\mathrm{H}(9 \mathrm{C})$ & 0.9602 \\
\hline$C(10)-H(10 A)$ & 0.9601 \\
\hline$C(10)-H(10 B)$ & 0.9599 \\
\hline$C(10)-H(10 C)$ & 0.9600 \\
\hline$C(11)-H(11 A)$ & 0.9601 \\
\hline$C(11)-H(11 B)$ & 0.9600 \\
\hline$C(11)-H(11 C)$ & 0.9599 \\
\hline$C(12)-H(12 A)$ & 0.9600 \\
\hline$C(12)-H(12 B)$ & 0.9600 \\
\hline$C(12)-H(12 C)$ & 0.9600 \\
\hline$c(13)-c(15)$ & $1.536(5)$ \\
\hline$C(13)-C(14)$ & $1.540(5)$ \\
\hline$C(13)-C(16)$ & $1.541(4)$ \\
\hline$C(14)-H(14 A)$ & 0.9599 \\
\hline$C(14)-H(14 B)$ & 0.9600 \\
\hline$C(14)-H(14 C)$ & 0.9600 \\
\hline$C(15)-H(15 A)$ & 0.9602 \\
\hline$C(15)-H(15 B)$ & 0.9600 \\
\hline$C(15)-H(15 C)$ & 0.9600 \\
\hline$C(16)-H(16 A)$ & 0.9601 \\
\hline$C(16)-H(16 B)$ & 0.9600 \\
\hline$C(16)-H(16 C)$ & 0.9600 \\
\hline$C(17)-C(20)$ & $1.542(5)$ \\
\hline$C(17)-C(18)$ & $1.542(4)$ \\
\hline$c(17)-C(19)$ & $1.552(5)$ \\
\hline$C(18)-H(18 A)$ & 0.9599 \\
\hline$C(18)-H(18 B)$ & 0.9599 \\
\hline$C(18)-H(18 C)$ & 0.9600 \\
\hline$C(19)-H(19 A)$ & 0.9600 \\
\hline$C(19)-H(19 B)$ & 0.9600 \\
\hline$C(19)-H(19 C)$ & 0.9599 \\
\hline$C(20)-H(20 A)$ & 0.9598 \\
\hline$C(20)-H(20 B)$ & 0.9599 \\
\hline$C(20)-H(20 C)$ & 0.9600 \\
\hline$C(21)-H(21 A)$ & 0.9600 \\
\hline$C(21)-H(21 B)$ & 0.9601 \\
\hline$C(21)-H(21 C)$ & 0.9600 \\
\hline$C(22)-C(23)$ & $1.526(6)$ \\
\hline$C(22)-C(25)$ & $1.543(6)$ \\
\hline$C(22)-C(24)$ & $1.546(5)$ \\
\hline$C(23)-H(23 A)$ & 0.9600 \\
\hline$C(23)-H(23 B)$ & 0.9599 \\
\hline $\mathrm{C}(23)-\mathrm{H}(23 \mathrm{C})$ & 0.9599 \\
\hline $\mathrm{C}(24)-\mathrm{H}(24 \mathrm{~A})$ & 0.9601 \\
\hline$C(24)-H(24 B)$ & 0.9599 \\
\hline$C(24)-H(24 C)$ & 0.9600 \\
\hline$C(25)-H(25 A)$ & 0.9600 \\
\hline$C(25)-H(25 B)$ & 0.9600 \\
\hline$C(25)-H(25 C)$ & 0.9599 \\
\hline
\end{tabular}




\begin{tabular}{|c|c|}
\hline$C(26)-C(28)$ & $1.532(6)$ \\
\hline$C(26)-C(29)$ & $1.532(5)$ \\
\hline$C(26)-C(27)$ & $1.552(5)$ \\
\hline $\mathrm{C}(27)-\mathrm{H}(27 \mathrm{~A})$ & 0.9600 \\
\hline$C(27)-H(27 B)$ & 0.9598 \\
\hline $\mathrm{C}(27)-\mathrm{H}(27 \mathrm{C})$ & 0.9600 \\
\hline$C(28)-H(28 A)$ & 0.9600 \\
\hline$C(28)-H(28 B)$ & 0.9600 \\
\hline $\mathrm{C}(28)-\mathrm{H}(28 \mathrm{C})$ & 0.9599 \\
\hline$C(29)-H(29 A)$ & 0.9600 \\
\hline$C(29)-H(29 B)$ & 0.9601 \\
\hline $\mathrm{C}(29)-\mathrm{H}(29 \mathrm{C})$ & 0.9600 \\
\hline$C(30)-C(35)$ & $1.397(4)$ \\
\hline$C(30)-C(31)$ & $1.407(5)$ \\
\hline$c(31)-c(32)$ & $1.389(5)$ \\
\hline$C(31)-H(31)$ & 0.9300 \\
\hline$C(32)-C(33)$ & $1.388(5)$ \\
\hline $\mathrm{C}(32)-\mathrm{H}(32)$ & 0.9300 \\
\hline$C(33)-C(34)$ & $1.378(6)$ \\
\hline $\mathrm{C}(33)-\mathrm{H}(33)$ & 0.9300 \\
\hline$C(34)-C(35)$ & $1.395(5)$ \\
\hline $\mathrm{C}(34)-\mathrm{H}(34)$ & 0.9301 \\
\hline $\mathrm{C}(35)-\mathrm{H}(35)$ & 0.9300 \\
\hline$C(36)-C(40)$ & $1.428(4)$ \\
\hline$c(36)-C(37)$ & $1.445(4)$ \\
\hline$C(36)-C(41)$ & $1.501(4)$ \\
\hline$c(37)-c(38)$ & $1.440(4)$ \\
\hline$c(37)-C(42)$ & $1.490(4)$ \\
\hline$C(38)-C(39)$ & $1.441(4)$ \\
\hline$C(38)-C(43)$ & $1.490(4)$ \\
\hline$C(39)-C(40)$ & $1.429(4)$ \\
\hline$C(39)-C(44)$ & $1.500(4)$ \\
\hline$C(40)-C(45)$ & $1.511(4)$ \\
\hline $\mathrm{C}(41)-\mathrm{H}(41 \mathrm{~A})$ & 0.9601 \\
\hline$C(41)-H(41 B)$ & 0.9599 \\
\hline$C(41)-H(41 C)$ & 0.9599 \\
\hline$C(42)-H(42 A)$ & 0.9599 \\
\hline$C(42)-H(42 B)$ & 0.9601 \\
\hline$C(42)-H(42 C)$ & 0.9600 \\
\hline$C(43)-H(43 A)$ & 0.9599 \\
\hline$C(43)-H(43 B)$ & 0.9600 \\
\hline$C(43)-H(43 C)$ & 0.9600 \\
\hline$C(44)-H(44 A)$ & 0.9599 \\
\hline$C(44)-H(44 B)$ & 0.9600 \\
\hline$C(44)-H(44 C)$ & 0.9600 \\
\hline$C(45)-H(45 A)$ & 0.9600 \\
\hline$C(45)-H(45 B)$ & 0.9600 \\
\hline$C(45)-H(45 C)$ & 0.9600 \\
\hline
\end{tabular}




\begin{tabular}{|c|c|}
\hline$C(1)-G e(1)-S i(1)$ & $98.71(9)$ \\
\hline$C(1)-G e(1)-S i(5)$ & $122.85(9)$ \\
\hline $\operatorname{Si}(1)-\mathrm{Ge}(1)-\mathrm{Si}(5)$ & $138.44(3)$ \\
\hline $\mathrm{C}(1)-\mathrm{Ge}(1)-\mathrm{Fe}(1)$ & $56.33(9)$ \\
\hline $\operatorname{Si}(1)-\mathrm{Ge}(1)-\mathrm{Fe}(1)$ & $61.20(2)$ \\
\hline $\mathrm{Si}(5)-\mathrm{Ge}(1)-\mathrm{Fe}(1)$ & $141.37(3)$ \\
\hline$C(36)-\mathrm{Fe}(1)-\mathrm{C}(37)$ & $40.48(12)$ \\
\hline $\mathrm{C}(36)-\mathrm{Fe}(1)-\mathrm{C}(38)$ & $67.63(12)$ \\
\hline $\mathrm{C}(37)-\mathrm{Fe}(1)-\mathrm{C}(38)$ & $40.23(12)$ \\
\hline$C(36)-\mathrm{Fe}(1)-\mathrm{C}(40)$ & $39.87(12)$ \\
\hline$C(37)-\mathrm{Fe}(1)-\mathrm{C}(40)$ & $67.27(12)$ \\
\hline $\mathrm{C}(38)-\mathrm{Fe}(1)-\mathrm{C}(40)$ & $67.03(12)$ \\
\hline$C(36)-\mathrm{Fe}(1)-\mathrm{C}(2)$ & $169.75(12)$ \\
\hline $\mathrm{C}(37)-\mathrm{Fe}(1)-\mathrm{C}(2)$ & $132.76(12)$ \\
\hline$C(38)-\mathrm{Fe}(1)-\mathrm{C}(2)$ & $102.45(12)$ \\
\hline$C(40)-\mathrm{Fe}(1)-\mathrm{C}(2)$ & $135.04(12)$ \\
\hline $\mathrm{C}(36)-\mathrm{Fe}(1)-\mathrm{C}(39)$ & $67.11(12)$ \\
\hline $\mathrm{C}(37)-\mathrm{Fe}(1)-\mathrm{C}(39)$ & $67.32(12)$ \\
\hline$C(38)-\mathrm{Fe}(1)-\mathrm{C}(39)$ & $40.03(12)$ \\
\hline$C(40)-\mathrm{Fe}(1)-\mathrm{C}(39)$ & $39.60(11)$ \\
\hline$C(2)-\mathrm{Fe}(1)-\mathrm{C}(39)$ & $103.80(12)$ \\
\hline$C(36)-\mathrm{Fe}(1)-\mathrm{C}(1)$ & $144.45(12)$ \\
\hline$C(37)-\mathrm{Fe}(1)-\mathrm{C}(1)$ & $163.36(12)$ \\
\hline$C(38)-\mathrm{Fe}(1)-\mathrm{C}(1)$ & $123.14(12)$ \\
\hline$C(40)-F e(1)-C(1)$ & $108.77(12)$ \\
\hline$C(2)-F e(1)-C(1)$ & $38.66(11)$ \\
\hline$C(39)-\mathrm{Fe}(1)-\mathrm{C}(1)$ & $99.01(12)$ \\
\hline $\mathrm{C}(36)-\mathrm{Fe}(1)-\mathrm{Si}(2)$ & $136.78(9)$ \\
\hline $\mathrm{C}(37)-\mathrm{Fe}(1)-\mathrm{Si}(2)$ & $105.17(9)$ \\
\hline $\mathrm{C}(38)-\mathrm{Fe}(1)-\mathrm{Si}(2)$ & $103.94(9)$ \\
\hline $\mathrm{C}(40)-\mathrm{Fe}(1)-\mathrm{Si}(2)$ & $170.87(9)$ \\
\hline$C(2)-F e(1)-S i(2)$ & $46.20(8)$ \\
\hline$C(39)-\mathrm{Fe}(1)-\operatorname{Si}(2)$ & $133.70(9)$ \\
\hline $\mathrm{C}(1)-\mathrm{Fe}(1)-\mathrm{Si}(2)$ & $76.94(8)$ \\
\hline$C(36)-\mathrm{Fe}(1)-\mathrm{Si}(1)$ & $103.75(9)$ \\
\hline $\mathrm{C}(37)-\mathrm{Fe}(1)-\mathrm{Si}(1)$ & $107.19(9)$ \\
\hline$C(38)-F e(1)-S i(1)$ & $139.60(9)$ \\
\hline$C(40)-\mathrm{Fe}(1)-\mathrm{Si}(1)$ & $131.88(9)$ \\
\hline $\mathrm{C}(2)-\mathrm{Fe}(1)-\mathrm{Si}(1)$ & $85.46(8)$ \\
\hline$C(39)-\mathrm{Fe}(1)-\mathrm{Si}(1)$ & $170.69(9)$ \\
\hline$C(1)-F e(1)-S i(1)$ & $87.57(8)$ \\
\hline $\operatorname{Si}(2)-\operatorname{Fe}(1)-\operatorname{Si}(1)$ & $54.13(3)$ \\
\hline$C(36)-\mathrm{Fe}(1)-\mathrm{Ge}(1)$ & $113.03(9)$ \\
\hline $\mathrm{C}(37)-\mathrm{Fe}(1)-\mathrm{Ge}(1)$ & $148.29(9)$ \\
\hline $\mathrm{C}(38)-\mathrm{Fe}(1)-\mathrm{Ge}(1)$ & $165.54(9)$ \\
\hline$C(40)-\mathrm{Fe}(1)-\mathrm{Ge}(1)$ & $103.72(9)$ \\
\hline$C(2)-\mathrm{Fe}(1)-\mathrm{Ge}(1)$ & $75.87(8)$ \\
\hline $\mathrm{C}(39)-\mathrm{Fe}(1)-\mathrm{Ge}(1)$ & $125.91(9)$ \\
\hline$C(1)-\mathrm{Fe}(1)-\mathrm{Ge}(1)$ & $47.55(8)$ \\
\hline $\mathrm{Si}(2)-\mathrm{Fe}(1)-\mathrm{Ge}(1)$ & $85.40(2)$ \\
\hline $\mathrm{Si}(1)-\mathrm{Fe}(1)-\mathrm{Ge}(1)$ & $54.85(2)$ \\
\hline
\end{tabular}


Si(2)-Si(1)-Ge(1)

Si(2)-Si(1)-Si(3)

$\mathrm{Ge}(1)-\mathrm{Si}(1)-\mathrm{Si}(3)$

$\mathrm{Si}(2)-\mathrm{Si}(1)-\mathrm{Fe}(1)$

$\mathrm{Ge}(1)-\mathrm{Si}(1)-\mathrm{Fe}$ (1)

$\mathrm{Si}(3)-\mathrm{Si}(1)-\mathrm{Fe}(1)$

$\mathrm{C}(2)-\mathrm{Si}(2)-\mathrm{Si}(1)$

$\mathrm{C}(2)-\mathrm{Si}(2)-\mathrm{Si}(4)$

Si(1)-Si(2)-Si(4)

$\mathrm{C}(2)-\mathrm{Si}(2)-\mathrm{Fe}(1)$

$\mathrm{Si}(1)-\mathrm{Si}(2)-\mathrm{Fe}(1)$

$\mathrm{Si}(4)-\mathrm{Si}(2)-\mathrm{Fe}(1)$

$\mathrm{C}(3)-\mathrm{Si}(3)-\mathrm{C}(8)$

$\mathrm{C}(3)-\mathrm{Si}(3)-\mathrm{C}(4)$

$\mathrm{C}(8)-\mathrm{Si}(3)-\mathrm{C}(4)$

$\mathrm{C}(3)-\mathrm{Si}(3)-\mathrm{Si}(1)$

$\mathrm{C}(8)-\mathrm{Si}(3)-\mathrm{Si}(1)$

$\mathrm{C}(4)-\mathrm{Si}(3)-\mathrm{Si}(1)$

$\mathrm{C}(12)-\mathrm{Si}(4)-\mathrm{C}(13)$

$\mathrm{C}(12)-\mathrm{Si}(4)-\mathrm{C}(17)$

$\mathrm{C}(13)-\mathrm{Si}(4)-\mathrm{C}(17)$

C(12)-Si(4)-Si(2)

C(13)-Si(4)-Si(2)

$\mathrm{C}(17)-\mathrm{Si}(4)-\mathrm{Si}(2)$

$\mathrm{C}(21)-\mathrm{Si}(5)-\mathrm{C}(26)$

$\mathrm{C}(21)-\mathrm{Si}(5)-\mathrm{C}(22)$

$\mathrm{C}(26)-\mathrm{Si}(5)-\mathrm{C}(22)$

$\mathrm{C}(21)-\mathrm{Si}(5)-\mathrm{Ge}(1)$

$\mathrm{C}(26)-\mathrm{Si}(5)-\mathrm{Ge}(1)$

$\mathrm{C}(22)-\mathrm{Si}(5)-\mathrm{Ge}(1)$

$\mathrm{C}(2)-\mathrm{C}(1)-\mathrm{C}(30)$

$\mathrm{C}(2)-\mathrm{C}(1)-\mathrm{Ge}(1)$

$\mathrm{C}(30)-\mathrm{C}(1)-\mathrm{Ge}(1)$

$\mathrm{C}(2)-\mathrm{C}(1)-\mathrm{Fe}(1)$

$\mathrm{C}(30)-\mathrm{C}(1)-\mathrm{Fe}(1)$

$\mathrm{Ge}(1)-\mathrm{C}(1)-\mathrm{Fe}(1)$

$\mathrm{C}(1)-\mathrm{C}(2)-\mathrm{Si}(2)$

$\mathrm{C}(1)-\mathrm{C}(2)-\mathrm{Fe}(1)$

$\mathrm{Si}(2)-\mathrm{C}(2)-\mathrm{Fe}(1)$

$\mathrm{C}(1)-\mathrm{C}(2)-\mathrm{H}(2)$

$\mathrm{Si}(2)-\mathrm{C}(2)-\mathrm{H}(2)$

$\mathrm{Fe}(1)-\mathrm{C}(2)-\mathrm{H}(2)$

$\mathrm{Si}(3)-C(3)-H(3 A)$

$\mathrm{Si}(3)-\mathrm{C}(3)-\mathrm{H}(3 \mathrm{~B})$

$\mathrm{H}(3 \mathrm{~A})-\mathrm{C}(3)-\mathrm{H}(3 \mathrm{~B})$

$\mathrm{Si}(3)-\mathrm{C}(3)-\mathrm{H}(3 \mathrm{C})$

$\mathrm{H}(3 \mathrm{~A})-\mathrm{C}(3)-\mathrm{H}(3 \mathrm{C})$

$\mathrm{H}(3 \mathrm{~B})-\mathrm{C}(3)-\mathrm{H}(3 \mathrm{C})$

$C(7)-C(4)-C(5)$

$C(7)-C(4)-C(6)$

$C(5)-C(4)-C(6)$
$96.34(4)$

$129.82(5)$

$129.47(4)$

$62.91(3)$

$63.95(3)$

$149.05(5)$

$99.49(10)$

$106.56(10)$

$152.91(4)$

$56.62(9)$

$62.96(3)$

$139.51(4)$

$105.24(17)$

$108.18(17)$

$114.42(16)$

$111.67(12)$

$111.24(11)$

$106.16(12)$

$106.77(15)$

$106.96(15)$

$115.38(14)$

$108.84(10)$

$116.39(11)$

$102.06(10)$

$104.96(18)$

$107.4(2)$

$115.42(17)$

$117.28(13)$

$109.03(12)$

$103.20(12)$

$118.8(3)$

$117.8(2)$

$123.1(2)$

$68.52(17)$

$130.2(2)$

$76.12(11)$

$125.7(2)$

$.72 .82(17)$

$77.18(11)$

117.1

117.2

124.5

109.5

109.5

109.5

109.4

109.5

109.5

$108.4(3)$

$109.3(3)$

$107.4(3)$ 


\begin{tabular}{|c|c|}
\hline$C(7)-C(4)-S i(3)$ & $111.4(2)$ \\
\hline$C(5)-C(4)-S i(3)$ & $109.9(3)$ \\
\hline$C(6)-C(4)-S i(3)$ & $110.3(3)$ \\
\hline$C(4)-C(5)-H(5 A)$ & 109.5 \\
\hline$C(4)-C(5)-H(5 B)$ & 109.5 \\
\hline$H(5 A)-C(5)-H(5 B)$ & 109.5 \\
\hline$C(4)-C(5)-H(5 C)$ & 109.4 \\
\hline$H(5 A)-C(5)-H(5 C)$ & 109.5 \\
\hline$H(5 B)-C(5)-H(5 C)$ & 109.5 \\
\hline$C(4)-C(6)-H(6 A)$ & 109.4 \\
\hline$C(4)-C(6)-H(6 B)$ & 109.5 \\
\hline$H(6 A)-C(6)-H(6 B)$ & 109.5 \\
\hline$C(4)-C(6)-H(6 C)$ & 109.5 \\
\hline$H(6 A)-C(6)-H(6 C)$ & 109.5 \\
\hline$H(6 B)-C(6)-H(6 C)$ & 109.5 \\
\hline$C(4)-C(7)-H(7 A)$ & 109.4 \\
\hline$C(4)-C(7)-H(7 B)$ & 109.5 \\
\hline$H(7 A)-C(7)-H(7 B)$ & 109.5 \\
\hline$C(4)-C(7)-H(7 C)$ & 109.4 \\
\hline$H(7 A)-C(7)-H(7 C)$ & 109.5 \\
\hline$H(7 B)-C(7)-H(7 C)$ & 109.5 \\
\hline$C(10)-C(8)-C(9)$ & $109.2(3)$ \\
\hline$C(10)-C(8)-C(11)$ & $108.5(3)$ \\
\hline$C(9)-C(8)-C(11)$ & $107.0(3)$ \\
\hline$c(10)-C(8)-S i(3)$ & $112.0(2)$ \\
\hline$C(9)-C(8)-S i(3)$ & $112.4(2)$ \\
\hline$C(11)-C(8)-S i(3)$ & $107.4(2)$ \\
\hline$C(8)-C(9)-H(9 A)$ & 109.5 \\
\hline$C(8)-C(9)-H(9 B)$ & 109.5 \\
\hline$H(9 A)-C(9)-H(9 B)$ & 109.5 \\
\hline$C(8)-C(9)-H(9 C)$ & 109.4 \\
\hline$H(9 A)-C(9)-H(9 C)$ & 109.5 \\
\hline$H(9 B)-C(9)-H(9 C)$ & 109.5 \\
\hline$C(8)-C(10)-H(10 A)$ & 109.6 \\
\hline$C(8)-C(10)-H(10 B)$ & 109.4 \\
\hline$H(10 A)-C(10)-H(10 B)$ & 109.5 \\
\hline$C(8)-C(10)-H(10 C)$ & 109.4 \\
\hline$H(10 A)-C(10)-H(10 C)$ & 109.5 \\
\hline $\mathrm{H}(10 \mathrm{~B})-\mathrm{C}(10)-\mathrm{H}(10 \mathrm{C})$ & 109.5 \\
\hline$C(8)-C(11)-H(11 A)$ & 109.5 \\
\hline$C(8)-C(11)-H(11 B)$ & 109.5 \\
\hline$H(11 A)-C(11)-H(11 B)$ & 109.5 \\
\hline$C(8)-C(11)-H(11 C)$ & 109.4 \\
\hline$H(11 A)-C(11)-H(11 C)$ & 109.5 \\
\hline $\mathrm{H}(11 \mathrm{~B})-\mathrm{C}(11)-\mathrm{H}(11 \mathrm{C})$ & 109.5 \\
\hline $\operatorname{Si}(4)-C(12)-H(12 A)$ & 109.5 \\
\hline $\mathrm{Si}(4)-C(12)-\mathrm{H}(12 \mathrm{~B})$ & 109.5 \\
\hline $\mathrm{H}(12 \mathrm{~A})-\mathrm{C}(12)-\mathrm{H}(12 \mathrm{~B})$ & 109.5 \\
\hline $\mathrm{Si}(4)-\mathrm{C}(12)-\mathrm{H}(12 \mathrm{C})$ & 109.5 \\
\hline $\mathrm{H}(12 \mathrm{~A})-\mathrm{C}(12)-\mathrm{H}(12 \mathrm{C})$ & 109.5 \\
\hline $\mathrm{H}(12 \mathrm{~B})-\mathrm{C}(12)-\mathrm{H}(12 \mathrm{C})$ & 109.5 \\
\hline
\end{tabular}


$C(15)-C(13)-C(14)$

$C(15)-C(13)-C(16)$

$C(14)-C(13)-C(16)$

$\mathrm{C}(15)-\mathrm{C}(13)-\mathrm{Si}(4)$

$\mathrm{C}(14)-\mathrm{C}(13)-\mathrm{Si}(4)$

$\mathrm{C}(16)-\mathrm{C}(13)-\mathrm{Si}(4)$

$\mathrm{C}(13)-\mathrm{C}(14)-\mathrm{H}(14 \mathrm{~A})$

$\mathrm{C}(13)-\mathrm{C}(14)-\mathrm{H}(14 \mathrm{~B})$

$\mathrm{H}(14 \mathrm{~A})-\mathrm{C}(14)-\mathrm{H}(14 \mathrm{~B})$

$\mathrm{C}(13)-\mathrm{C}(14)-\mathrm{H}(14 \mathrm{C})$

$\mathrm{H}(14 \mathrm{~A})-\mathrm{C}(14)-\mathrm{H}(14 \mathrm{C})$

$\mathrm{H}(14 \mathrm{~B})-\mathrm{C}(14)-\mathrm{H}(14 \mathrm{C})$

$C(13)-C(15)-H(15 A)$

$C(13)-C(15)-H(15 B)$

$\mathrm{H}(15 \mathrm{~A})-\mathrm{C}(15)-\mathrm{H}(15 \mathrm{~B})$

$\mathrm{C}(13)-\mathrm{C}(15)-\mathrm{H}(15 \mathrm{C})$

$\mathrm{H}(15 \mathrm{~A})-\mathrm{C}(15)-\mathrm{H}(15 \mathrm{C})$

$\mathrm{H}(15 \mathrm{~B})-\mathrm{C}(15)-\mathrm{H}(15 \mathrm{C})$

$\mathrm{C}(13)-\mathrm{C}(16)-\mathrm{H}(16 \mathrm{~A})$

$C(13)-C(16)-H(16 B)$

$\mathrm{H}(16 \mathrm{~A})-\mathrm{C}(16)-\mathrm{H}(16 \mathrm{~B})$

$\mathrm{C}(13)-\mathrm{C}(16)-\mathrm{H}(16 \mathrm{C})$

$\mathrm{H}(16 \mathrm{~A})-\mathrm{C}(16)-\mathrm{H}(16 \mathrm{C})$

$\mathrm{H}(16 \mathrm{~B})-\mathrm{C}(16)-\mathrm{H}(16 \mathrm{C})$

$\mathrm{C}(20)-\mathrm{C}(17)-\mathrm{C}(18)$

$C(20)-C(17)-C(19)$

$C(18)-C(17)-C(19)$

$\mathrm{C}(20)-\mathrm{C}(17)-\mathrm{Si}(4)$

$\mathrm{C}(18)-\mathrm{C}(17)-\mathrm{Si}(4)$

$\mathrm{C}(19)-\mathrm{C}(17)-\mathrm{Si}(4)$

$\mathrm{C}(17)-\mathrm{C}(18)-\mathrm{H}(18 \mathrm{~A})$

$\mathrm{C}(17)-\mathrm{C}(18)-\mathrm{H}(18 \mathrm{~B})$

$\mathrm{H}(18 \mathrm{~A})-\mathrm{C}(18)-\mathrm{H}(18 \mathrm{~B})$

$\mathrm{C}(17)-\mathrm{C}(18)-\mathrm{H}(18 \mathrm{C})$

$\mathrm{H}(18 \mathrm{~A})-\mathrm{C}(18)-\mathrm{H}(18 \mathrm{C})$

$\mathrm{H}(18 \mathrm{~B})-\mathrm{C}(18)-\mathrm{H}(18 \mathrm{C})$

$\mathrm{C}(17)-\mathrm{C}(19)-\mathrm{H}(19 \mathrm{~A})$

$\mathrm{C}(17)-\mathrm{C}(19)-\mathrm{H}(19 \mathrm{~B})$

$\mathrm{H}(19 \mathrm{~A})-\mathrm{C}(19)-\mathrm{H}(19 \mathrm{~B})$

$\mathrm{C}(17)-\mathrm{C}(19)-\mathrm{H}(19 \mathrm{C})$

$\mathrm{H}(19 \mathrm{~A})-\mathrm{C}(19)-\mathrm{H}(19 \mathrm{C})$

$\mathrm{H}(19 \mathrm{~B})-\mathrm{C}(19)-\mathrm{H}(19 \mathrm{C})$

$\mathrm{C}(17)-\mathrm{C}(20)-\mathrm{H}(20 \mathrm{~A})$

$\mathrm{C}(17)-\mathrm{C}(20)-\mathrm{H}(20 \mathrm{~B})$

$\mathrm{H}(20 \mathrm{~A})-\mathrm{C}(20)-\mathrm{H}(20 \mathrm{~B})$

$\mathrm{C}(17)-\mathrm{C}(20)-\mathrm{H}(20 \mathrm{C})$

$\mathrm{H}(20 \mathrm{~A})-\mathrm{C}(20)-\mathrm{H}(20 \mathrm{C})$

$\mathrm{H}(20 \mathrm{~B})-\mathrm{C}(20)-\mathrm{H}(20 \mathrm{C})$

$\mathrm{Si}(5)-\mathrm{C}(21)-\mathrm{H}(21 \mathrm{~A})$

$\mathrm{Si}(5)-\mathrm{C}(21)-\mathrm{H}(21 \mathrm{~B})$

$\mathrm{H}(21 \mathrm{~A})-\mathrm{C}(21)-\mathrm{H}(21 \mathrm{~B})$
$109.0(3)$

$108.7(3)$

$108.0(3)$

$112.2(2)$

$108.2(2)$

$110.6(2)$

109.4

109.5

109.5

109.5

109.5

109.5

109.4

109.5

109.5

109.5

109.5

109.5

109.4

109.6

109.5

109.5

109.5

109.5

$107.9(3)$

$107.3(3)$

$108.2(3)$

$109.2(2)$

$112.5(2)$

$111.6(2)$

109.4

109.6

109.5

109.4

109.5

109.5

109.5

109.5

109.5

109.5

109.5

109.5

109.5

109.4

109.5

109.6

109.5

109.5

109.5

109.5

109.5 


\begin{tabular}{|c|c|}
\hline $\mathrm{Si}(5)-\mathrm{C}(21)-\mathrm{H}(21 \mathrm{C})$ & 109.4 \\
\hline$H(21 A)-C(21)-H(21 C)$ & 109.5 \\
\hline $\mathrm{H}(21 \mathrm{~B})-\mathrm{C}(21)-\mathrm{H}(21 \mathrm{C})$ & 109.5 \\
\hline$C(23)-C(22)-C(25)$ & $108.0(4)$ \\
\hline$C(23)-C(22)-C(24)$ & $108.9(4)$ \\
\hline$C(25)-C(22)-C(24)$ & $108.0(4)$ \\
\hline$C(23)-C(22)-S i(5)$ & $107.9(3)$ \\
\hline$C(25)-C(22)-S i(5)$ & $113.6(3)$ \\
\hline$C(24)-C(22)-S i(5)$ & $110.4(3)$ \\
\hline$C(22)-C(23)-H(23 A)$ & 109.3 \\
\hline$C(22)-C(23)-H(23 B)$ & 109.5 \\
\hline $\mathrm{H}(23 \mathrm{~A})-\mathrm{C}(23)-\mathrm{H}(23 \mathrm{~B})$ & 109.5 \\
\hline$C(22)-C(23)-H(23 C)$ & 109.5 \\
\hline $\mathrm{H}(23 \mathrm{~A})-\mathrm{C}(23)-\mathrm{H}(23 \mathrm{C})$ & 109.5 \\
\hline$H(23 B)-C(23)-H(23 C)$ & 109.5 \\
\hline$C(22)-C(24)-H(24 A)$ & 109.5 \\
\hline$C(22)-C(24)-H(24 B)$ & 109.4 \\
\hline $\mathrm{H}(24 \mathrm{~A})-\mathrm{C}(24)-\mathrm{H}(24 \mathrm{~B})$ & 109.5 \\
\hline$C(22)-C(24)-H(24 C)$ & 109.6 \\
\hline$H(24 A)-C(24)-H(24 C)$ & 109.5 \\
\hline $\mathrm{H}(24 \mathrm{~B})-\mathrm{C}(24)-\mathrm{H}(24 \mathrm{C})$ & 109.5 \\
\hline$C(22)-C(25)-H(25 A)$ & 109.4 \\
\hline$C(22)-C(25)-H(25 B)$ & 109.5 \\
\hline$H(25 A)-C(25)-H(25 B)$ & 109.5 \\
\hline$C(22)-C(25)-H(25 C)$ & 109.5 \\
\hline$H(25 A)-C(25)-H(25 C)$ & 109.5 \\
\hline $\mathrm{H}(25 \mathrm{~B})-\mathrm{C}(25)-\mathrm{H}(25 \mathrm{C})$ & 109.5 \\
\hline$C(28)-C(26)-C(29)$ & $109.5(3)$ \\
\hline$C(28)-C(26)-C(27)$ & $107.4(3)$ \\
\hline$C(29)-C(26)-C(27)$ & $108.0(4)$ \\
\hline$C(28)-C(26)-S i(5)$ & $111.6(3)$ \\
\hline$C(29)-C(26)-S i(5)$ & $109.0(3)$ \\
\hline$C(27)-C(26)-S i(5)$ & $111.2(3)$ \\
\hline$C(26)-C(27)-H(27 A)$ & 109.3 \\
\hline$C(26)-C(27)-H(27 B)$ & 109.6 \\
\hline$H(27 A)-C(27)-H(27 B)$ & 109.5 \\
\hline$C(26)-C(27)-H(27 C)$ & 109.6 \\
\hline $\mathrm{H}(27 \mathrm{~A})-\mathrm{C}(27)-\mathrm{H}(27 \mathrm{C})$ & 109.5 \\
\hline $\mathrm{H}(27 \mathrm{~B})-\mathrm{C}(27)-\mathrm{H}(27 \mathrm{C})$ & 109.5 \\
\hline$C(26)-C(28)-H(28 A)$ & 109.4 \\
\hline$C(26)-C(28)-H(28 B)$ & 109.5 \\
\hline$H(28 A)-C(28)-H(28 B)$ & 109.5 \\
\hline$C(26)-C(28)-H(28 C)$ & 109.5 \\
\hline$H(28 A)-C(28)-H(28 C)$ & 109.5 \\
\hline$H(28 B)-C(28)-H(28 C)$ & 109.5 \\
\hline$C(26)-C(29)-H(29 A)$ & 109.4 \\
\hline$C(26)-C(29)-H(29 B)$ & 109.5 \\
\hline$H(29 A)-C(29)-H(29 B)$ & 109.5 \\
\hline$C(26)-C(29)-H(29 C)$ & 109.5 \\
\hline$H(29 A)-C(29)-H(29 C)$ & 109.5 \\
\hline$H(29 B)-C(29)-H(29 C)$ & 109.5 \\
\hline
\end{tabular}


$C(35)-C(30)-C(31)$

$116.8(3)$

$C(35)-C(30)-C(1)$

$122.9(3)$

$\mathrm{C}(31)-\mathrm{C}(30)-\mathrm{C}(1)$

$120.3(3)$

$C(32)-C(31)-C(30)$

$121.7(3)$

$\mathrm{C}(32)-\mathrm{C}(31)-\mathrm{H}(31)$

119.1

$\mathrm{C}(30)-\mathrm{C}(31)-\mathrm{H}(31)$

119.2

$\mathrm{C}(33)-\mathrm{C}(32)-\mathrm{C}(31)$

$120.1(3)$

$\mathrm{C}(33)-\mathrm{C}(32)-\mathrm{H}(32)$

119.9

$\mathrm{C}(31)-\mathrm{C}(32)-\mathrm{H}(32)$

119.9

$\mathrm{C}(34)-\mathrm{C}(33)-\mathrm{C}(32)$

$\mathrm{C}(34)-\mathrm{C}(33)-\mathrm{H}(33)$

$\mathrm{C}(32)-\mathrm{C}(33)-\mathrm{H}(33)$

$\mathrm{C}(33)-\mathrm{C}(34)-\mathrm{C}(35)$

$\mathrm{C}(33)-\mathrm{C}(34)-\mathrm{H}(34)$

$\mathrm{C}(35)-\mathrm{C}(34)-\mathrm{H}(34)$

$\mathrm{C}(34)-\mathrm{C}(35)-\mathrm{C}(30)$

$\mathrm{C}(34)-\mathrm{C}(35)-\mathrm{H}(35)$

$\mathrm{C}(30)-\mathrm{C}(35)-\mathrm{H}(35)$

$\mathrm{C}(40)-\mathrm{C}(36)-\mathrm{C}(37)$

$\mathrm{C}(40)-\mathrm{C}(36)-\mathrm{C}(41)$

$119.3(3)$

120.3

120.4

$120.6(3)$

119.7

119.7

$121.5(3)$

119.2

119.3

$108.0(3)$

$124.7(3)$

$127.0(3)$

$70.73(18)$

$70.05(18)$

$\mathrm{C}(40)-\mathrm{C}(36)-\mathrm{Fe}(1)$

$\mathrm{C}(37)-\mathrm{C}(36)-\mathrm{Fe}(1)$

$130.4(2)$

$\mathrm{C}(41)-\mathrm{C}(36)-\mathrm{Fe}(1)$

$\mathrm{C}(38)-\mathrm{C}(37)-\mathrm{C}(36)$

$107.4(3)$

$\mathrm{C}(38)-\mathrm{C}(37)-\mathrm{C}(42)$

$124.7(3)$

$C(36)-C(37)-C(42)$

$127.4(3)$

$\mathrm{C}(38)-\mathrm{C}(37)-\mathrm{Fe}(1)$

$69.94(18)$

$\mathrm{C}(36)-\mathrm{C}(37)-\mathrm{Fe}(1)$

$69.48(18)$

$\mathrm{C}(42)-\mathrm{C}(37)-\mathrm{Fe}(1)$

$132.3(2)$

$\mathrm{C}(37)-\mathrm{C}(38)-\mathrm{C}(39)$

$108.1(3)$

$\mathrm{C}(37)-\mathrm{C}(38)-\mathrm{C}(43)$

$126.6(3)$

$\mathrm{C}(39)-\mathrm{C}(38)-\mathrm{C}(43)$

$124.8(3)$

$\mathrm{C}(37)-\mathrm{C}(38)-\mathrm{Fe}(1)$

$69.84(18)$

$\mathrm{C}(39)-\mathrm{C}(38)-\mathrm{Fe}(1)$

$70.80(17)$

$\mathrm{C}(43)-\mathrm{C}(38)-\mathrm{Fe}(1)$

$130.8(2)$

$\mathrm{C}(40)-\mathrm{C}(39)-\mathrm{C}(38)$

$107.7(3)$

$C(40)-C(39)-C(44)$

$128.4(3)$

$\mathrm{C}(38)-\mathrm{C}(39)-\mathrm{C}(44)$

$\mathrm{C}(40)-\mathrm{C}(39)-\mathrm{Fe}(1)$

$123.7(3)$

$69.71(17)$

$\mathrm{C}(38)-\mathrm{C}(39)-\mathrm{Fe}(1)$

$69.18(17)$

$\mathrm{C}(44)-\mathrm{C}(39)-\mathrm{Fe}(1)$

$\mathrm{C}(36)-\mathrm{C}(40)-\mathrm{C}(39)$

$C(36)-C(40)-C(45)$

$\mathrm{C}(39)-\mathrm{C}(40)-\mathrm{C}(45)$

$\mathrm{C}(36)-\mathrm{C}(40)-\mathrm{Fe}(1)$

$\mathrm{C}(39)-\mathrm{C}(40)-\mathrm{Fe}(1)$

$\mathrm{C}(45)-\mathrm{C}(40)-\mathrm{Fe}(1)$

$\mathrm{C}(36)-\mathrm{C}(41)-\mathrm{H}(41 \mathrm{~A})$

$C(36)-C(41)-H(41 B)$

$130.5(2)$

$108.7(3)$

$124.0(3)$

$126.4(3)$

$69.40(17)$

$70.69(17)$

$133.9(2)$

109.3

109.5

$\mathrm{H}(41 \mathrm{~A})-\mathrm{C}(41)-\mathrm{H}(41 \mathrm{~B})$

109.5 


\begin{tabular}{ll}
$\mathrm{C}(36)-\mathrm{C}(41)-\mathrm{H}(41 \mathrm{C})$ & 109.6 \\
$\mathrm{H}(41 \mathrm{~A})-\mathrm{C}(41)-\mathrm{H}(41 \mathrm{C})$ & 109.5 \\
$\mathrm{H}(41 \mathrm{~B})-\mathrm{C}(41)-\mathrm{H}(41 \mathrm{C})$ & 109.5 \\
$\mathrm{C}(37)-\mathrm{C}(42)-\mathrm{H}(42 \mathrm{~A})$ & 109.5 \\
$\mathrm{C}(37)-\mathrm{C}(42)-\mathrm{H}(42 \mathrm{~B})$ & 109.4 \\
$\mathrm{H}(42 \mathrm{~A})-\mathrm{C}(42)-\mathrm{H}(42 \mathrm{~B})$ & 109.5 \\
$\mathrm{C}(37)-\mathrm{C}(42)-\mathrm{H}(42 \mathrm{C})$ & 109.5 \\
$\mathrm{H}(42 \mathrm{~A})-\mathrm{C}(42)-\mathrm{H}(42 \mathrm{C})$ & 109.5 \\
$\mathrm{H}(42 \mathrm{~B})-\mathrm{C}(42)-\mathrm{H}(42 \mathrm{C})$ & 109.5 \\
$\mathrm{C}(38)-\mathrm{C}(43)-\mathrm{H}(43 \mathrm{~A})$ & 109.4 \\
$\mathrm{C}(38)-\mathrm{C}(43)-\mathrm{H}(43 \mathrm{~B})$ & 109.5 \\
$\mathrm{H}(43 \mathrm{~A})-\mathrm{C}(43)-\mathrm{H}(43 \mathrm{~B})$ & 109.5 \\
$\mathrm{C}(38)-\mathrm{C}(43)-\mathrm{H}(43 \mathrm{C})$ & 109.5 \\
$\mathrm{H}(43 \mathrm{~A})-\mathrm{C}(43)-\mathrm{H}(43 \mathrm{C})$ & 109.5 \\
$\mathrm{H}(43 \mathrm{~B})-\mathrm{C}(43)-\mathrm{H}(43 \mathrm{C})$ & 109.5 \\
$\mathrm{C}(39)-\mathrm{C}(44)-\mathrm{H}(44 \mathrm{~A})$ & 109.5 \\
$\mathrm{C}(39)-\mathrm{C}(44)-\mathrm{H}(44 \mathrm{~B})$ & 109.5 \\
$\mathrm{H}(44 \mathrm{~A})-\mathrm{C}(44)-\mathrm{H}(44 \mathrm{~B})$ & 109.5 \\
$\mathrm{C}(39)-\mathrm{C}(44)-\mathrm{H}(44 \mathrm{C})$ & 109.4 \\
$\mathrm{H}(44 \mathrm{~A})-\mathrm{C}(44)-\mathrm{H}(44 \mathrm{C})$ & 109.5 \\
$\mathrm{H}(44 \mathrm{~B})-\mathrm{C}(44)-\mathrm{H}(44 \mathrm{C})$ & 109.5 \\
$\mathrm{C}(40)-\mathrm{C}(45)-\mathrm{H}(45 \mathrm{~A})$ & 109.3 \\
$\mathrm{C}(40)-\mathrm{C}(45)-\mathrm{H}(45 \mathrm{~B})$ & 109.5 \\
$\mathrm{H}(45 \mathrm{~A})-\mathrm{C}(45)-\mathrm{H}(45 \mathrm{~B})$ & 109.5 \\
$\mathrm{C}(40)-\mathrm{C}(45)-\mathrm{H}(45 \mathrm{C})$ & 109.5 \\
$\mathrm{H}(45 \mathrm{~A})-\mathrm{C}(45)-\mathrm{H}(45 \mathrm{C})$ & 109.5 \\
$\mathrm{H}(45 \mathrm{~B})-\mathrm{C}(45)-\mathrm{H}(45 \mathrm{C})$ & 109.5 \\
& \\
\hline
\end{tabular}

Symmetry transformations used to generate equivalent atoms: 
Table 4. Anisotropic displacement parameters $\left(A^{\wedge} 2 \times 10^{\wedge} 3\right)$ for 2 . The anisotropic displacement factor exponent takes the form:

$-2 \mathrm{pi}^{\wedge} 2$ [ $\mathrm{h}^{\wedge} 2 \mathrm{a}^{* \wedge} \mathrm{U} 11+\ldots+2 \mathrm{hk} \mathrm{a}^{*} \mathrm{~b}^{*} \mathrm{U} 12$ ]

\begin{tabular}{|c|c|c|c|c|c|c|}
\hline & $\mathrm{U} 11$ & $\mathrm{U} 22$ & U33 & U23 & $\mathrm{U} 13$ & U12 \\
\hline $\mathrm{Ge}(1)$ & $29(1)$ & $25(1)$ & $21(1)$ & $1(1)$ & $-1(1)$ & $0(1)$ \\
\hline $\mathrm{Fe}(1)$ & $20(1)$ & $15(1)$ & $17(1)$ & $-2(1)$ & $-1(1)$ & $-2(1)$ \\
\hline $\operatorname{si(1)}$ & $23(1)$ & $22(1)$ & $18(1)$ & $-3(1)$ & $1(1)$ & $1(1)$ \\
\hline $\operatorname{si(2)}$ & $14(1)$ & $7(1)$ & $12(1)$ & $1(1)$ & $0(1)$ & $1(1)$ \\
\hline $\operatorname{si(3)}$ & $26(1)$ & $23(1)$ & $21(1)$ & $-4(1)$ & $1(1)$ & $2(1)$ \\
\hline $\operatorname{si}(4)$ & $23(1)$ & $14(1)$ & $19(1)$ & $-1(1)$ & $-1(1)$ & $-1(1)$ \\
\hline $\operatorname{si}(5)$ & $29(1)$ & $28(1)$ & $22(1)$ & $4(1)$ & $0(1)$ & $1(1)$ \\
\hline$c(1)$ & $20(2)$ & $16(1)$ & $24(2)$ & $-3(1)$ & $0(1)$ & $-2(1)$ \\
\hline$c(2)$ & $18(1)$ & $17(1)$ & $18(1)$ & $-1(1)$ & $-1(1)$ & $-3(1)$ \\
\hline$C(3)$ & $35(2)$ & $35(2)$ & $30(2)$ & $2(1)$ & $8(1)$ & $3(2)$ \\
\hline$C(4)$ & $36(2)$ & $30(2)$ & $27(2)$ & $-10(1)$ & $-1(1)$ & $-2(2)$ \\
\hline$C(5)$ & $37(2)$ & $44(2)$ & $44(2)$ & $-15(2)$ & $-9(2)$ & $-2(2)$ \\
\hline$c(6)$ & $47(3)$ & $53(3)$ & $39(2)$ & $-25(2)$ & $-5(2)$ & $2(2)$ \\
\hline$c(7)$ & $52(3)$ & $31(2)$ & $43(2)$ & $-7(2)$ & $-8(2)$ & $-13(2)$ \\
\hline$c(8)$ & $24(2)$ & $30(2)$ & $28(2)$ & $-4(1)$ & $1(1)$ & $1(1)$ \\
\hline$C(9)$ & $34(2)$ & $35(2)$ & $36(2)$ & $-7(2)$ & $-2(2)$ & $11(2)$ \\
\hline$c(10)$ & $30(2)$ & $36(2)$ & $26(2)$ & $-2(1)$ & $-3(1)$ & $2(2)$ \\
\hline$c(11)$ & $29(2)$ & $40(2)$ & $43(2)$ & $-3(2)$ & $1(2)$ & $-5(2)$ \\
\hline$c(12)$ & $32(2)$ & $20(2)$ & $24(2)$ & $-4(1)$ & $3(1)$ & $-4(1)$ \\
\hline$c(13)$ & $28(2)$ & $17(1)$ & $24(2)$ & $1(1)$ & $-3(1)$ & $1(1)$ \\
\hline$c(14)$ & $28(2)$ & $26(2)$ & $39(2)$ & $1(1)$ & $-10(1)$ & $-2(1)$ \\
\hline$c(15)$ & $32(2)$ & $24(2)$ & $37(2)$ & $-6(1)$ & $2(1)$ & $1(1)$ \\
\hline$c(16)$ & $42(2)$ & $24(2)$ & $27(2)$ & $8(1)$ & $-3(1)$ & $-1(2)$ \\
\hline$c(17)$ & $25(2)$ & $18(1)$ & $27(2)$ & $-3(1)$ & $0(1)$ & $-2(1)$ \\
\hline$C(18)$ & $30(2)$ & $32(2)$ & $32(2)$ & $-10(1)$ & $-3(1)$ & $-7(1)$ \\
\hline$C(19)$ & $33(2)$ & $25(2)$ & $42(2)$ & $-4(1)$ & $4(2)$ & $-8(1)$ \\
\hline$C(20)$ & $24(2)$ & $27(2)$ & $46(2)$ & $-5(2)$ & $-2(1)$ & $-1(1)$ \\
\hline$C(21)$ & $57(3)$ & $30(2)$ & $32(2)$ & $5(2)$ & $1(2)$ & $7(2)$ \\
\hline$c(22)$ & $32(2)$ & $48(2)$ & $25(2)$ & $5(2)$ & $-7(1)$ & $-6(2)$ \\
\hline$c(23)$ & $31(2)$ & $100(4)$ & $40(2)$ & $-11(3)$ & $2(2)$ & $-11(2)$ \\
\hline$C(24)$ & $47(3)$ & $68(3)$ & $38(2)$ & $8(2)$ & $-16(2)$ & $-4(2)$ \\
\hline$c(25)$ & $46(3)$ & $53(3)$ & $59(3)$ & $-3(2)$ & $-9(2)$ & $-14(2)$ \\
\hline$c(26)$ & $35(2)$ & $39(2)$ & $25(2)$ & $5(2)$ & $3(1)$ & $-2(2)$ \\
\hline$c(27)$ & $57(3)$ & $50(3)$ & $34(2)$ & $18(2)$ & $13(2)$ & $-1(2)$ \\
\hline$c(28)$ & $45(2)$ & $52(3)$ & $37(2)$ & $-10(2)$ & $5(2)$ & $4(2)$ \\
\hline$C(29)$ & $33(2)$ & $54(3)$ & $37(2)$ & $1(2)$ & $5(2)$ & $-10(2)$ \\
\hline$C(30)$ & $22(2)$ & $18(1)$ & $24(2)$ & $-4(1)$ & $-5(1)$ & $2(1)$ \\
\hline$C(31)$ & $22(2)$ & $22(2)$ & $27(2)$ & $-6(1)$ & $-3(1)$ & $0(1)$ \\
\hline$c(32)$ & $23(2)$ & $34(2)$ & $36(2)$ & $-14(2)$ & $-1(1)$ & $0(1)$ \\
\hline$c(33)$ & $31(2)$ & $31(2)$ & $40(2)$ & $-14(2)$ & $-8(1)$ & $11(2)$ \\
\hline$c(34)$ & $42(2)$ & $21(2)$ & $35(2)$ & $-2(1)$ & $-8(2)$ & $6(2)$ \\
\hline
\end{tabular}




$\begin{array}{lllllrr}C(35) & 29(2) & 20(2) & 33(2) & -2(1) & -2(1) & -1(1) \\ C(36) & 25(2) & 22(2) & 22(2) & -3(1) & -2(1) & -9(1) \\ C(37) & 24(2) & 20(1) & 24(2) & -4(1) & -2(1) & -5(1) \\ C(38) & 26(2) & 22(2) & 23(2) & -5(1) & -3(1) & -7(1) \\ C(39) & 28(2) & 19(1) & 19(1) & -5(1) & -2(1) & -6(1) \\ C(40) & 28(2) & 18(1) & 21(2) & -3(1) & -1(1) & -6(1) \\ C(41) & 27(2) & 32(2) & 28(2) & -4(1) & 5(1) & -10(1) \\ C(42) & 26(2) & 27(2) & 34(2) & -3(1) & -6(1) & -3(1) \\ C(43) & 35(2) & 28(2) & 26(2) & -4(1) & -5(1) & -8(1) \\ C(44) & 37(2) & 25(2) & 25(2) & -8(1) & 2(1) & -3(1) \\ C(45) & 41(2) & 17(2) & 29(2) & 0(1) & 0(1) & -7(1) \\ \end{array}$


Table 5. Hydrogen coordinates $\left(x 10^{\wedge} 4\right)$ and isotropic displacement parameters $\left(A^{\wedge} 2 \times 10^{\wedge} 3\right)$ for 2 .

\begin{tabular}{|c|c|c|c|c|}
\hline & $x$ & $y$ & $z$ & $\mathrm{U}(\mathrm{eq})$ \\
\hline $\mathrm{H}(2)$ & 507 & 5521 & 3750 & 21 \\
\hline $\mathrm{H}(3 \mathrm{~A})$ & 4340 & 5028 & 780 & 42 \\
\hline $\mathrm{H}(3 \mathrm{~B})$ & 5703 & 4192 & 745 & 42 \\
\hline$H(3 C)$ & 5610 & 5208 & 1140 & 42 \\
\hline$H(5 A)$ & 2193 & 3885 & 889 & 49 \\
\hline$H(5 B)$ & 1604 & 3564 & 1549 & 49 \\
\hline $\mathrm{H}(5 \mathrm{C})$ & 1579 & 2742 & 1048 & 49 \\
\hline$H(6 A)$ & 5084 & 1759 & 950 & 54 \\
\hline $\mathrm{H}(6 \mathrm{~B})$ & 4295 & 2764 & 517 & 54 \\
\hline $\mathrm{H}(6 \mathrm{C})$ & 3665 & 1633 & 698 & 54 \\
\hline $\mathrm{H}(7 \mathrm{~A})$ & 4161 & 1270 & 2035 & 49 \\
\hline $\mathrm{H}(7 \mathrm{~B})$ & 2776 & 1149 & 1748 & 49 \\
\hline $\mathrm{H}(7 \mathrm{C})$ & 2806 & 1971 & 2249 & 49 \\
\hline $\mathrm{H}(9 \mathrm{~A})$ & 6500 & 1503 & 1704 & 44 \\
\hline $\mathrm{H}(9 \mathrm{~B})$ & 7852 & 1807 & 1945 & 44 \\
\hline $\mathrm{H}(9 \mathrm{C})$ & 7237 & 2458 & 1332 & 44 \\
\hline $\mathrm{H}(10 \mathrm{~A})$ & 5357 & 1868 & 2735 & 38 \\
\hline $\mathrm{H}(10 \mathrm{~B})$ & 5439 & 3031 & 2984 & 38 \\
\hline $\mathrm{H}(10 \mathrm{C})$ & 6754 & 2144 & 2941 & 38 \\
\hline$H(11 A)$ & 7278 & 4305 & 1728 & 45 \\
\hline $\mathrm{H}(11 \mathrm{~B})$ & 7926 & 3631 & 2329 & 45 \\
\hline $\mathrm{H}(1 \mathrm{C})$ & 6612 & 4523 & 2369 & 45 \\
\hline$H(12 A)$ & 482 & 4136 & 4716 & 31 \\
\hline $\mathrm{H}(12 \mathrm{~B})$ & 1987 & 3861 & 4939 & 31 \\
\hline $\mathrm{H}(12 \mathrm{C})$ & 957 & 2993 & 5132 & 31 \\
\hline $\mathrm{H}(14 \mathrm{~A})$ & 4281 & 2718 & 4727 & 37 \\
\hline$H(14 B)$ & 4725 & 2635 & 4040 & 37 \\
\hline $\mathrm{H}(14 \mathrm{C})$ & 5232 & 1637 & 4549 & 37 \\
\hline$H(15 A)$ & 2782 & 462 & 3778 & 38 \\
\hline$H(15 B)$ & 4312 & 256 & 3965 & 38 \\
\hline $\mathrm{H}(15 \mathrm{C})$ & 3800 & 1251 & 3457 & 38 \\
\hline$H(16 A)$ & 2695 & 1430 & 5250 & 39 \\
\hline$H(16 B)$ & 3676 & 378 & 5057 & 39 \\
\hline $\mathrm{H}(16 \mathrm{C})$ & 2135 & 547 & 4890 & 39 \\
\hline $\mathrm{H}(18 \mathrm{~A})$ & 1044 & 1351 & 3163 & 36 \\
\hline $\mathrm{H}(18 \mathrm{~B})$ & 474 & 2603 & 2899 & 36 \\
\hline $\mathrm{H}(18 \mathrm{C})$ & -523 & 1699 & 3069 & 36 \\
\hline$H(19 A)$ & 489 & 632 & 4279 & 40 \\
\hline $\mathrm{H}(19 \mathrm{~B})$ & -1050 & 1012 & 4127 & 40 \\
\hline$H(19 C)$ & -421 & 1458 & 4678 & 40 \\
\hline$H(20 A)$ & -1199 & 3478 & 4251 & 39 \\
\hline $\mathrm{H}(20 \mathrm{~B})$ & -1886 & 2984 & 3731 & 39 \\
\hline
\end{tabular}




\begin{tabular}{|c|c|c|c|c|}
\hline $\mathrm{H}(20 \mathrm{C})$ & -899 & 3896 & 3561 & 39 \\
\hline$H(21 A)$ & -407 & 9349 & 1653 & 50 \\
\hline $\mathrm{H}(21 \mathrm{~B})$ & 58 & 9727 & 978 & 50 \\
\hline $\mathrm{H}(21 \mathrm{C})$ & 1107 & 9540 & 1508 & 50 \\
\hline $\mathrm{H}(23 \mathrm{~A})$ & -2073 & 8203 & 1543 & 68 \\
\hline$H(23 B)$ & -1655 & 6949 & 1850 & 68 \\
\hline $\mathrm{H}(23 \mathrm{C})$ & -2781 & 7211 & 1352 & 68 \\
\hline $\mathrm{H}(24 A)$ & -694 & 7806 & 51 & 63 \\
\hline $\mathrm{H}(24 \mathrm{~B})$ & -1515 & 8726 & 423 & 63 \\
\hline $\mathrm{H}(24 \mathrm{C})$ & -2201 & 7699 & 261 & 63 \\
\hline $\mathrm{H}(25 \mathrm{~A})$ & 10 & 5814 & 567 & 63 \\
\hline $\mathrm{H}(25 \mathrm{~B})$ & -1514 & 5753 & 764 & 63 \\
\hline $\mathrm{H}(25 \mathrm{C})$ & -380 & 5496 & 1259 & 63 \\
\hline$H(27 A)$ & 1183 & 8763 & -67 & 60 \\
\hline $\mathrm{H}(27 \mathrm{~B})$ & 2761 & 8740 & -152 & 60 \\
\hline $\mathrm{H}(27 \mathrm{C})$ & 1961 & 9452 & 333 & 60 \\
\hline$H(28 A)$ & 1547 & 6627 & 96 & 54 \\
\hline$H(28 B)$ & 2568 & 5977 & 590 & 54 \\
\hline $\mathrm{H}(28 \mathrm{C})$ & 3111 & 6671 & 1 & 54 \\
\hline$H(29 A)$ & 3485 & 8413 & 1165 & 50 \\
\hline$H(29 B)$ & 4304 & 7768 & 659 & 50 \\
\hline$H(29 C)$ & 3760 & 7076 & 1249 & 50 \\
\hline $\mathrm{H}(31)$ & -1641 & 6350 & 3604 & 28 \\
\hline $\mathrm{H}(32)$ & -3404 & 7748 & 3800 & 37 \\
\hline $\mathrm{H}(33)$ & -3344 & 9630 & 3386 & 41 \\
\hline H (34) & -1542 & 10082 & 2753 & 40 \\
\hline $\mathrm{H}(35)$ & 232 & 8689 & 2555 & 33 \\
\hline$H(41 A)$ & 6194 & 6345 & 2515 & 34 \\
\hline $\mathrm{H}(41 \mathrm{~B})$ & 5973 & 7679 & 2486 & 34 \\
\hline $\mathrm{H}(41 \mathrm{C})$ & 5063 & 7076 & 2099 & 34 \\
\hline$H(42 A)$ & 6347 & 5071 & 3341 & 35 \\
\hline$H(42 B)$ & 5355 & 4428 & 3783 & 35 \\
\hline $\mathrm{H}(42 \mathrm{C})$ & 6213 & 5269 & 4034 & 35 \\
\hline $\mathrm{H}(43 \mathrm{~A})$ & 3884 & 5165 & 4657 & 35 \\
\hline $\mathrm{H}(43 \mathrm{~B})$ & 2344 & 5685 & 4620 & 35 \\
\hline $\mathrm{H}(43 \mathrm{C})$ & 3397 & 6362 & 4874 & 35 \\
\hline $\mathrm{H}(44 \mathrm{~A})$ & 1401 & 7715 & 4382 & 35 \\
\hline $\mathrm{H}(44 \mathrm{~B})$ & 822 & 8279 & 3745 & 35 \\
\hline $\mathrm{H}(44 \mathrm{C})$ & 1869 & 8856 & 4072 & 35 \\
\hline$H(45 A)$ & 2287 & 9339 & 2846 & 35 \\
\hline$H(45 B)$ & 2963 & 8771 & 2283 & 35 \\
\hline $\mathrm{H}(45 \mathrm{C})$ & 3846 & 9332 & 2703 & 35 \\
\hline
\end{tabular}

